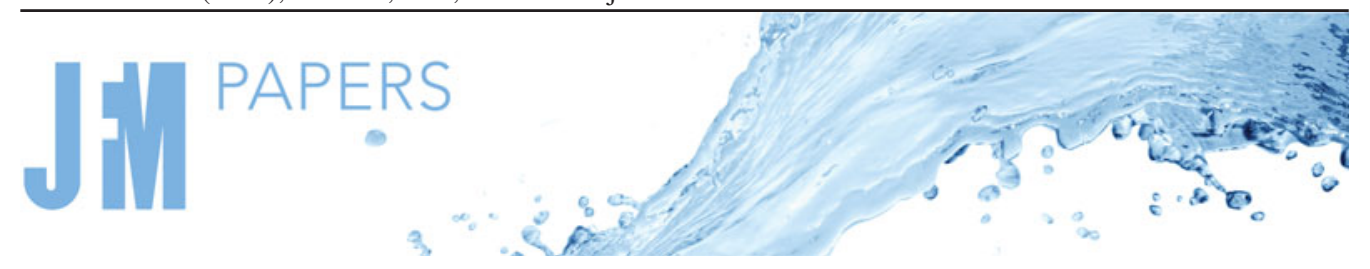

\title{
Lubricated gravity currents of power-law fluids
}

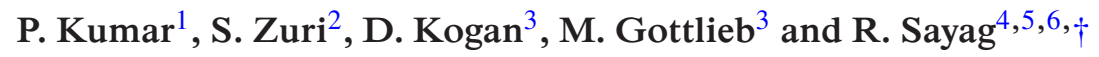 \\ ${ }^{1}$ Ashoka University, Rajiv Gandhi Education City, Haryana 131029, India \\ ${ }^{2}$ Schulich Faculty of Chemistry, Technion, Haifa 3200003, Israel \\ ${ }^{3}$ Department of Chemical Engineering, Ben-Gurion University of the Negev, Beer-Sheva 8410501, Israel \\ ${ }^{4}$ Department of Environmental Physics, Blaustein Institutes for Desert Research, Ben-Gurion University \\ of the Negev, Sde Boker 8499000, Israel \\ ${ }^{5}$ Department of Mechanical Engineering, Ben-Gurion University of the Negev, Beer-Sheva 8410501, Israel \\ ${ }^{6}$ Department of Physics, Ben-Gurion University of the Negev, Beer-Sheva 8410501, Israel
}

(Received 14 October 2020; revised 16 January 2021; accepted 12 March 2021)

The motion of glaciers over their bedrock or drops of fluid along a solid surface can vary dramatically when these substrates are lubricated. We investigate the coupled flow of a gravity current (GC) of a strain-rate softening fluid that is lubricated by a denser, lower-viscosity Newtonian fluid. We present a set of experiments in which such GCs are discharged axisymmetrically and at constant flux over a flat surface. Using imaging techniques, we follow the evolution of the front and thickness field of each fluid. We find that, unlike purely Newtonian lubricated GCs, the fronts of the non-Newtonian and lubricating Newtonian fluids have a power-law time evolution with different exponents. Each of these exponents is similar to that of a non-lubricated GC of the same fluid. Nevertheless, the fronts of our lubricated GCs evolve faster than those of the corresponding non-lubricated GCs owing to larger intercepts. In addition, in contrast with the monotonically declining thickness of non-lubricated GCs, the thickness of the lubricated, non-Newtonian fluid is nearly uniform, and that of the lubricating fluid is non-monotonic with localised spikes. Despite these complex thickness patterns, lubricated GCs remain axisymmetric as long as the flux of the lubricating fluid is sufficiently smaller than that of the non-Newtonian fluid.

Key words: complex fluids, geophysical and geological flows, interfacial flows (free surface)

$\dagger$ Email address for correspondence: roiy@bgu.ac.il

(C) The Author(s), 2021. Published by Cambridge University Press. This is an Open Access article, distributed under the terms of the Creative Commons Attribution licence (http://creativecommons.org/ licenses/by/4.0/), which permits unrestricted re-use, distribution, and reproduction in any medium, provided the original work is properly cited. 


\section{P. Kumar, S. Zuri, D. Kogan, M. Gottlieb and R. Sayag}

\section{Introduction}

Gravity-driven flows of one fluid over another can involve complex interactions between the two fluids, which can lead to a rich dynamic behaviour. Such flows occur in a wide range of natural and human-made systems, as in lava flow over less viscous lava (Balmforth et al. 2000; Griffiths 2000), spreading of the lithosphere over the mid-mantle boundary (Lister \& Kerr 1989; Dauck et al. 2019), ice flow over an ocean (Kivelson et al. 2000; DeConto \& Pollard 2016) and over bedrock covered with sediment and water (Fowler 1987; Stokes et al. 2007), flows in porous media (Woods \& Mason 2000), and droplet motion on liquid-infused surfaces (Keiser et al. 2017).

The flow of GCs in a circular geometry has been studied with a range of boundary conditions. In the absence of a lubricating layer, a common boundary condition along the base of a sole GC is no slip. Such GCs of Newtonian fluids that are discharged at constant flux follow a similarity solution, in which the front position at time $t$ is proportional to $t^{1 / 2}$ (Huppert 1982). Similar GCs of power-law (PL) fluids having exponent $n$, where $n=1$ represents a Newtonian fluid and $n>1$ represents a strain-rate softening fluid, also have similarity solutions, in which the front propagation is proportional to $t^{(2 n+2) /(5 n+3)}$ (Sayag \& Worster 2013).

At the other extreme, the presence of a lower fluid layer can significantly reduce friction at the base of the top fluid, which results in extensionally-dominated GCs. This is the case, for example, for ice shelves, which deform over the relatively inviscid oceans with weak friction along their interface. The late-time front evolution of such axisymmetric GCs of Newtonian fluids is proportional to $t$ (Pegler \& Worster 2012). However, when the top fluid is strain-rate softening, an initially axisymmetric front can destabilise and develop fingering patterns that consist of tongues separated by rifts (Sayag \& Worster 2019).

In the more general case, friction along the boundaries of the GCs can vary spatiotemporally as their stress field evolves. For example, the interface of an ice sheet with its underlying bed rock can include distributed melt water and sediments, which impose non-uniform and time-dependent friction along the ice base, and evolve spatiotemporally under the stresses imposed by the ice layer (Fowler 1981; Schoof \& Hewitt 2013). Consequently, the coupled ice-lubricant system may evolve various flow patterns, such as ice streams (Stokes et al. 2007) and glacier surges (Fowler 1987). Such systems have been modelled as two coupled GCs of Newtonian fluids spreading one on top of the other (Kowal \& Worster 2015). The early stage of these flows follows a self-similar evolution, in which the fronts of the two fluids evolve like $t^{1 / 2}$, as in non-lubricated (no-slip) GCs, and they can have a radially non-monotonic thickness. However, experiments showed that, at a later stage, these coupled flows became unstable and developed fingering patterns and non-axisymmetric flow (Kowal \& Worster 2015). It has been suggested that such instabilities appear when the jump in hydrostatic pressure gradient across the lubrication front is negative (Kowal \& Worster 2019).

Despite the wide range of natural lubricated GCs that involve non-Newtonian fluids, the radial flow of a non-Newtonian fluid over a lubricated layer of a Newtonian fluid has not been explored thus far. In this study, we begin to explore such flows by experimentally investigating the flow of a strain-rate softening fluid that is lubricated by a Newtonian fluid, in a similar setting as in Kowal \& Worster (2015). We focus on flows that are axisymmetric to the leading order, and explore the case where the flux and viscosity of the lubricating fluid are much smaller than those of the non-Newtonian top fluid. We trace the evolution of the two fronts, and the spatiotemporal evolution of the light transmitted through the two fluids, from which we resolve the thickness distribution of the 
(a)

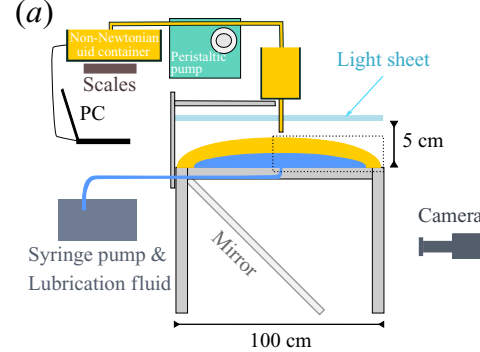

(b)

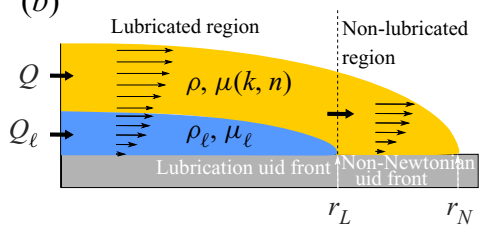

$(c)$

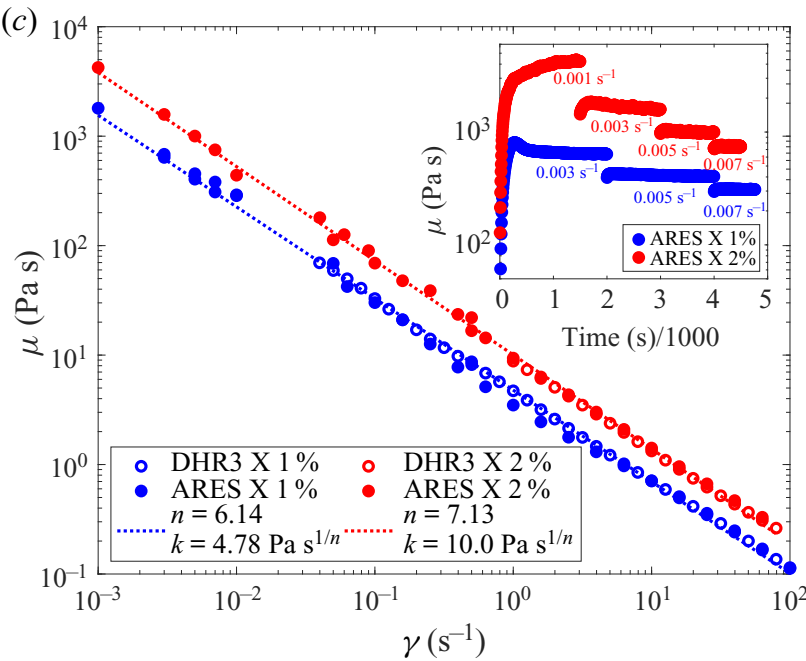

Figure 1. (a) Our experimental apparatus for lubricated GCs. (b) Close-up of the flow region (dash-line rectangle in $(a)$ ). (c) Viscosity measurements as a function of strain rate $(\bullet, \circ)$ of $1 \%$ (blue) and $2 \%$ (red) of xanthan solutions and the regression to power-law functions $(\cdots)$. Inset shows two sets of typical viscosity measurements for each fluid concentration, in which the strain rate varies between 0.001 and $0.007 \mathrm{~s}^{-1}$ in steps of $0.002 \mathrm{~s}^{-1}$, and at each step, the viscosity is measured continuously until an equilibrium value is reached.

top-layer, non-Newtonian fluid. We then contrast our findings with the present theories of non-lubricated and lubricated GCs.

\section{Experimental set-up}

The experimental apparatus (figure $1 a, b$ ) included a flat, optically-transparent square glass sheet of $1 \times 1 \mathrm{~m}^{2}$ and a thickness of $10 \mathrm{~mm}$, supported by an aluminum frame parallel to the ground with an alignment accuracy of 50 micron $\mathrm{m}^{-1}$. A rectangular plane mirror was placed underneath the glass sheet at an angle of $45^{\circ}$ to the horizontal for imaging. The centre of the glass sheet had a $5 \mathrm{~mm}$ diameter nozzle that was connected to a syringe pump (NE4000) that delivered, at constant flux $Q_{\ell}$, the Newtonian lubricating fluid of viscosity $\mu_{\ell}$ and density $\rho_{\ell}$.

The non-Newtonian fluid of viscosity $\mu$ and density $\rho$ was driven at constant flux $Q$ by gravity from a beaker that was supported by an $x y z$-translation stage through an $8 \mathrm{~mm}$ diameter aluminum tube, whose outlet was $15 \mathrm{~mm}$ over the glass surface (figure $1 a, b$ ). We kept the flux constant by keeping a constant fluid level in the beaker using a peristaltic pump that supplied fluid from a reservoir. A $1 \times 1 \mathrm{~m}^{2}$ white light-sheet and a diffuser were positioned parallel to the glass surface and approximately $50 \mathrm{~mm}$ over it to illuminate the flow uniformly. A time-lapsed image sequence was captured throughout each experiment using a Nikon D5500 camera that was facing the $45^{\circ}$ mirror.

\subsection{Preparation and properties of the experimental fluids}

The non-Newtonian fluid we used was an aqueous solution of food-grade xanthan gum (Jungbunzlauer) of $1 \%$ and $2 \%$ concentration (per weight). To prepare uniformly-dissolved and air-bubble-free solutions, we followed the following procedure: first, we generated a smooth vortex (Eurostat-200) in a beaker containing deionised water. Then, we poured the xanthan-gum powder into the centre of the vortex within less than $60 \mathrm{~s}$, to ensure uniform 


\section{P. Kumar, S. Zuri, D. Kogan, M. Gottlieb and R. Sayag}

dispersion of the powder and prevent aggregates before the viscosity of the solution soared. After 90 min of mixing, we added $0.3 \mathrm{~g}$ of lemon-yellow colour per $5000 \mathrm{~g}$ of solution, and stirred for an additional $30 \mathrm{~min}$. Finally, we stored the solution in a $4{ }^{\circ} \mathrm{C}$ refrigerator for 24 $\mathrm{h}$ to remove residual air bubbles. The densities of the solutions were $1.0035 \pm 0.0005$ and $1.007 \pm 0.0005 \mathrm{~g} \mathrm{~cm}^{-3}$ for the $1 \%$ and $2 \%$ concentrations, respectively.

The rheology of xanthan gum solutions of such concentrations has been studied extensively in various flow configurations, and we have described it in detail in Sayag $\&$ Worster (2019). In general, such xanthan solutions are viscoelastic. However, in flows such as those we consider here, the role of elastic deformation is significantly smaller compared with viscous deformation (e.g. Sayag \& Worster 2013, 2019), as implied from the small Deborah number that we estimate in $\S 4$. Therefore, here we focus on the viscous deformation of our xanthan solutions, which is known to be consistent with a PL fluid of both shear and extensional thinning for a wide range of strain rates, with an approximately similar exponent (Martín-Alfonso et al. 2018).

We used TA DHR-3 and ARES rheometers to measure the dependence of the shear viscosity $\mu$ of our xanthan solutions on the shear rate $\dot{\epsilon}$. The setup consisted of a steady shear flow within a $2^{\circ}$ cone-and-plate geometry (40 mm (DHR-3) and $50 \mathrm{~mm}$ (ARES)) and a solvent trap to minimise dehydration. The rate of shear was varied in discrete increasing steps, and the duration of the viscosity measurements at each step was sufficiently long to ascertain that a steady value was reached within a $1 \%$ standard deviation (STD) (figure 1c, inset). We observed that the viscosity equilibration time was shorter when the shear-rate difference between two consecutive steps was smaller, and when the imposed shear rate was larger. At very low shear rates, the equilibration time was substantial but, at shear rates larger than $0.01 \mathrm{~s}^{-1}$, the equilibration time was negligible. Fitting our measured viscosity to the power-law relation

$$
\mu=k \dot{\epsilon}^{1 / n-1},
$$

while keeping the consistency $k$ and the exponent $n$ as free parameters, we find that

$$
\begin{aligned}
& n=6.14 \pm 0.19, \quad k=4.78 \pm 0.07 \mathrm{~Pa} \mathrm{~s}^{1 / n} \quad \text { for the } 1 \% \text { solution, } \\
& n=7.14 \pm 0.23, \quad k=10.05 \pm 0.13 \mathrm{~Pa} \mathrm{~s}^{1 / n} \quad \text { for the } 2 \% \text { solution, }
\end{aligned}
$$

where each uncertainty represents one STD in the measured quantity (figure $1 c$ ).

For a Newtonian lubricating fluid of larger density and lower viscosity, we dissolved glucose in distilled water $(\approx 38 \%$ glucose in weight) to obtain a solution of density $\rho_{\ell}=1.164 \pm 0.0005 \mathrm{~g} \mathrm{~cm}^{-3}$ and of dynamic viscosity $\mu_{\ell}=9.1 \pm 0.12 \mathrm{mPa}$ s at $20^{\circ} \mathrm{C}$. We dyed the lubricating fluid in blue $(0.5 \%$ per weight $)$ to achieve a high contrast of the evolving fluid-fluid interface and to more easily resolve the evolving thickness of the non-Newtonian fluid layer, as discussed in $\S 3.2$.

\subsection{Experimental procedure and preliminary experiments}

Two preparation procedures had a major impact on the reproducibility of our experiments. The first was the concentric alignment of the two outlet nozzles of the discharged fluids to an accuracy of $\pm 100 \mu \mathrm{m}$. This was done using an acrylic cylindrical adapter that had a circular cavity at one base, concentric with a circular protrusion at the opposite base, which respectively were fitted to the outer diameter of the tube delivering the non-Newtonian fluid and to the inner diameter of the nozzle in the glass surface that delivered the lubricating fluid. The adapter was initially placed over the glass substrate with its protrusion connected to the lower nozzle. Then the xyz-stage was lowered 
Lubricated gravity currents of power-law fluids

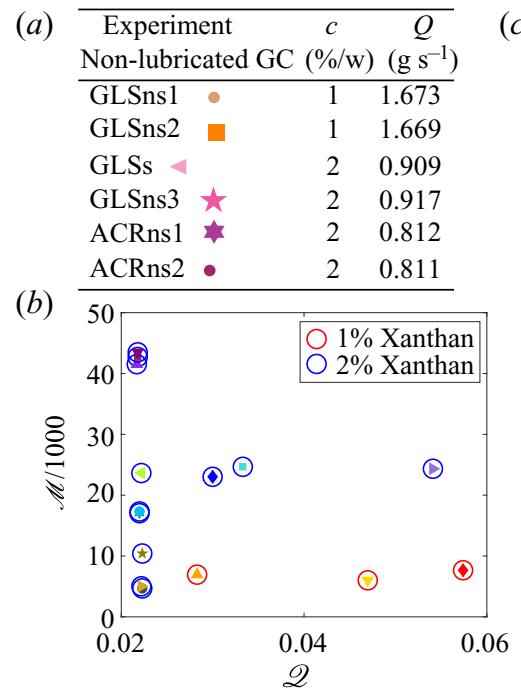

\begin{tabular}{|c|c|c|c|c|c|c|}
\hline $\begin{array}{l}\text { Experiment } \\
\text { Lubricated GC }\end{array}$ & $\mathscr{Q}$ & $\mathscr{M}$ & $\begin{array}{c}c \\
(\% / w)\end{array}$ & $\begin{array}{c}Q \\
\left(\mathrm{~g} \mathrm{~s}^{-1}\right)\end{array}$ & $\begin{array}{l}t_{L} \\
(\mathrm{~s})\end{array}$ & $\begin{array}{r}r_{N}\left(t_{L}\right) \\
(\mathrm{cm})\end{array}$ \\
\hline $1 \diamond$ & 0.0574 & 7661 & 1 & 1.167 & 798 & 20 \\
\hline $2 \Delta$ & 0.0283 & 6936 & 1 & 1.767 & 752 & 20 \\
\hline $3 \dagger \nabla$ & 0.04697 & 6006 & 1 & 1.767 & 594 & 20 \\
\hline $4 \uparrow \bullet$ & 0.0223 & 4671 & 2 & 4.716 & 78 & 10 \\
\hline 5 & 0.0218 & 42717 & 2 & 0.643 & 1550 & 20 \\
\hline $6>$ & 0.0222 & 5023 & 2 & 3.643 & 82 & 10 \\
\hline $7 \dagger<$ & 0.0222 & 23639 & 2 & 1.5 & 754 & 20 \\
\hline $8 \star$ & 0.0223 & 10412 & 2 & 1.525 & 208 & 10 \\
\hline $9 \star$ & 0.022 & 17036 & 2 & 1.5 & 450 & 15 \\
\hline $10 \bullet$ & 0.022 & 17323 & 2 & 1.5 & 462 & 15 \\
\hline 11 & 0.0333 & 24661 & 2 & 1.5 & 806 & 20 \\
\hline 12 & 0.03 & 23017 & 2 & 1.664 & 742 & 20 \\
\hline $13 \dagger \Delta$ & 0.0217 & 41528 & 2 & 0.692 & 1510 & 20 \\
\hline $14 \nabla$ & 0.0218 & 43483 & 2 & 0.643 & 1594 & 20 \\
\hline $15>$ & 0.0541 & 24343 & 2 & 1.533 & 794 & 20 \\
\hline
\end{tabular}

Figure 2. (a) The non-lubricated GC experiments were performed on glass (GLS) or acrylic (ACR) substrates with a pre-coat of soap solution (s) or without one (ns). (b) The lubricated GC experiments in the $\mathscr{M}-\mathscr{Q}$ state space. (c) The lubricated GC experiments. All experiments were performed at $22^{\circ} \mathrm{C}$.

( $z$ direction) so that the feeding tube of the non-Newtonian fluid approached the adapter, and was tuned horizontally ( $x-y$ directions) to fit the tube into the cavity in the adapter. The calibration was completed when the two nozzles were simultaneously connected to the adapter, at which point the stage was lifted ( $z$ direction) and the adapter was removed. The second procedure concerns the impact of the wetting conditions over the glass surface on the shape of the lubricating-fluid front. In the absence of the non-Newtonian fluid, the axisymmetry of the lubricating-fluid front was sensitive to the wetting conditions of the glass surface. We reduced this sensitivity to the level that the axisymmetric shape of the lubricating-fluid front persisted, by forming uniform wetting conditions over the glass surface using a coating of soap solution (3.3\% liquid dish soap in deionised water) that was allowed to dehydrate prior to the initiation of our experiments. To verify that the soap coating had no impact on the non-Newtonian GC, we compared the front evolution of a non-lubricated GCs with and without a soap coating, and found no measurable difference for xanthan concentrations of either $1 \%$ or $2 \%$ (figure $2 a$ ). A possible cause for the difference in sensitivity to the wetting conditions between the two fluids is the large $(\approx 16)$ Bond number of the non-Newtonian fluid and the relatively small $(\approx 0.03)$ Bond number of the lubricating Newtonian fluid. We elaborate on this in $\S 4$.

We conducted 15 lubricated GC experiments (figure $2 b, c$ ) in the following procedure. Initially, we released the non-Newtonian fluid axisymmetrically in a constant flux. When the evolving GC reached a radius of $10-20 \mathrm{~cm}\left(t=t_{L}\right)$, we initiated the axisymmetric discharge of the lubricating fluid underneath the non-Newtonian fluid (figure $3 a$, see supplementary movies 1-4 available at https://doi.org/10.1017/jfm.2021.240).

\section{Experimental analysis}

We classify the lubricated GC experiments using the four dimensionless numbers

$$
\mathscr{Q} \equiv \frac{Q_{\ell}}{Q}, \quad \mathscr{D} \equiv \frac{\rho_{\ell}-\rho}{\rho}, \quad n, \quad \mathscr{M} \equiv \frac{\mu}{\mu_{\ell}}=\frac{\rho g}{\mu_{\ell}}\left(\frac{k}{\rho g}\right)^{8 n /(5 n+3)}\left(\frac{t_{L}^{4}}{Q}\right)^{(n-1) /(5 n+3)},
$$


(a)
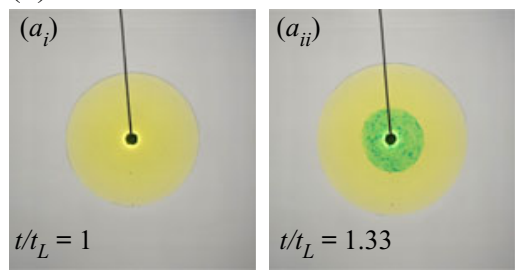

(b)

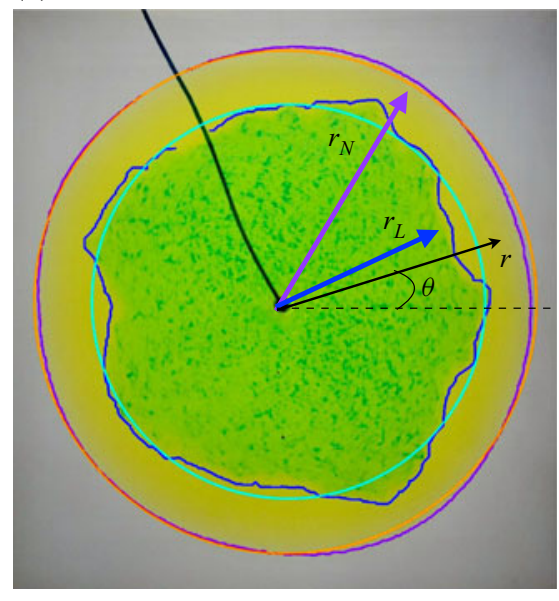

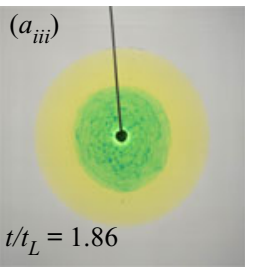
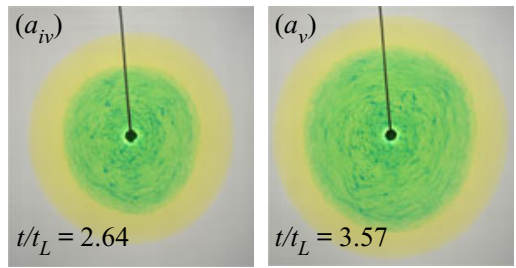

(c)

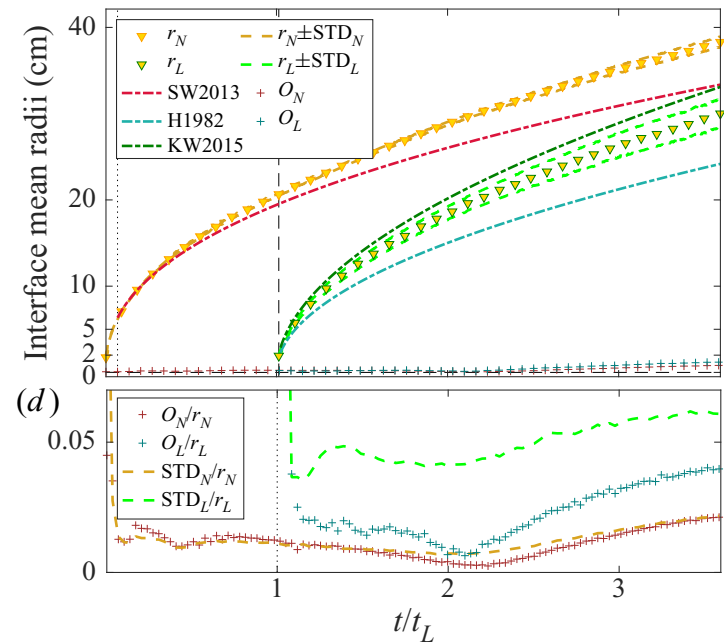

Figure 3. (a) Time series of snapshots from experiment no. 7, showing the xanthan solution (yellow) lubricated by a glucose solution (appears green) that started $t_{L}=754 \mathrm{~s}$ later. (b) A snapshot from experiment no. 3 , showing (using polar coordinates $(r, \theta)$ ) the measured front $r_{L}(\theta)$ of the lubricating fluid (- blue) and $r_{N}(\theta)$ of the non-Newtonian fluid (- purple), and the corresponding fitted circles in ( - , cyan) and $(-$, orange), respectively. (c) Evolution of the average (fitted) front radii $r_{N}(\nabla$, yellow) and the corresponding regression STD (---, yellow) of experiment no. 3 compared with the theoretical prediction of a non-lubricated GC of a PL fluid (Sayag \& Worster 2013) with identical fluid parameters (----, red). Similarly, for the lubricating fluid front, we show the average front radii $r_{L}(\nabla$, dark green) and $\operatorname{STD}(---$, green) compared with predictions for non-lubricated Newtonian GC (Huppert 1982) (----, teal) and lubricated purely Newtonian GC (Kowal \& Worster 2015) with exponent $\beta=0.52$ and coefficient $\xi_{L}^{*}=0.27$ (----, dark green). The centres of the fitted circles $O_{N}, O_{L}(+)$ remain very close to the centre of the nozzle (shown in more detail in panel $d$ ). $(d)$ STDs of the front measurements (---) and the centres of the fitted circles $O_{N}, O_{L}(+)$ normalised by the instantaneous average fronts $r_{N}, r_{L}$.

which represent respectively the sources flux ratio, the reduced density ratio, the PL fluid exponent, and the viscosity ratio. The non-Newtonian fluid viscosity in the latter ratio is based on (2.1) and on the solution of non-lubricated GCs of PL fluids with a small depth-to-radius aspect ratio (Sayag \& Worster 2013). Specifically, the leading-order shear rate in these GCs, $\dot{\epsilon}=|1 / 2 \partial u / \partial z|$, where $u$ is the radial velocity and $z$ is the depth coordinate, is scaled with the thickness and radius like $\dot{\epsilon} \sim(\rho g / k)^{n} H^{2 n} / R^{n}$, where $H$ and $R$ are the depth and radial scales, respectively. These spatial scales have a power-law evolution in time that is determined by a similarity solution (Sayag \& Worster 2013), which we evaluate at the lubricating fluid discharge time $t_{L}$ to obtain the viscosity scale in $(3.1 d)$. In the experiments that we analyse, the lubrication flux was much smaller than the flux of the PL fluid ( $\mathscr{Q} \lesssim 0.06$ ). The viscosity ratio varied over almost one order of magnitude (4700 $\lesssim \mathscr{M} \lesssim 43000$ ), particularly owing to the wide range of $t_{L}$ and $Q$ (figure $2 c$ ) and 


\section{Lubricated gravity currents of power-law fluids}

owing to the fluid exponent $n>1$, as represented by the last factor in the definition $(3.1 d)$ of $\mathscr{M}$. The $1 \%$ and $2 \%$ polymer concentrations led to a small variation in $n$ (figure $1 c$ ) and to similar density ratios of $\mathscr{D}_{1 \%}=0.156$ and $\mathscr{D}_{2} \%=0.152$, respectively.

To explore the significance of the interaction between the two fluids, we analyse in the sections that follow the image sequence taken in each experiment to trace the evolution of the two fluid fronts $(\$ 3.1)$ and thicknesses $(\$ 3.2)$, and we compare the resulting measurements to existing theories of lubricated and non-lubricated flows.

\subsection{The front evolution of the lubricating and non-Newtonian fluids}

Tracing the position of the fronts of the non-Newtonian fluid $r_{N}(t, \theta)$ and of the lubricating fluid $r_{L}(t, \theta)$, where $\theta$ is the angular coordinate, we calculate the average radius of each instantaneous front by fitting a circle (figure $3 b$ ). We find that the regression STD can be reduced when using the centres of the fitted circles $O_{N}$ and $O_{L}$ as free parameters in addition to the radii (figure $3 c, d$ ). This means that the deviation of the front from an axisymmetric shape has two contributions: the centres $O_{N}, O_{L}$ that represent translation of the geometric centre of the circular front, and the regression STD of the radii that represent the non-axisymmetric displacement of the fronts with respect to the translated circles. We find that $\mathrm{STD}_{L}, O_{L} \lesssim 5$ and $\mathrm{STD}_{N}, O_{N} \lesssim 2 \mathrm{~cm}$, which implies that the lubricating fluid became more non-axisymmetric and had larger variations in its geometric centre than the non-Newtonian fluid. However, the ratios $\operatorname{STD}_{i} / r_{i}$ and $O_{i} / r_{i}$, where the subscript $i$ represents the two fronts $N \& L$, remain confined (less than $\approx 5 \%$ ) and even decay (figure $3 d$ ), which implies that the evolution of the fluid fronts remained axisymmetric to the leading order.

To appreciate the resulting evolution of the fronts, we contrast them with existing theories of non-lubricated GCs of PL (Sayag \& Worster 2013) and Newtonian (Huppert 1982) fluids, and lubricated GCs of purely Newtonian fluids (Kowal \& Worster 2015), in which the solutions for the fronts are respectively

$$
\begin{gathered}
r_{N}^{\mathrm{SW} 13}=\xi_{N} b_{N} t^{(2 n+2) /(5 n+3)}, \quad b_{N}=\left[2^{1-n} Q^{2 n+1}(\rho g / k)^{n} /(n+2)\right]^{1 /(5 n+3)}, \\
r_{L}^{\mathrm{H} 82}=\xi_{L} b_{L} t^{1 / 2}, \quad b_{L}=\left(Q_{\ell}^{3}\left(\rho_{\ell}-\rho\right) g / 3 \mu_{\ell}\right)^{1 / 8}, \\
r_{L}^{\mathrm{KW} 15}=\xi_{L}^{*} b_{L}^{*} t^{\beta}, \quad b_{L}^{*}=\left(\mathscr{M} \mathscr{Q} Q^{3} \rho g / \mu\right)^{1 / 8}=\left(\mathscr{Q} Q^{3} \rho g / \mu_{\ell}\right)^{1 / 8},
\end{gathered}
$$

where for constant flux, $\xi_{N}=\xi_{L} \approx 0.71$ were determined theoretically (Huppert 1982; Sayag \& Worster 2013), and $\xi_{L}^{*} \approx 0.27 \pm 0.02, \beta=0.52 \pm 0.02$ were estimated experimentally for the purely Newtonian case in the range $1 / \mathscr{M} \ll \mathscr{Q} \ll 1$, which is consistent with the range we consider, though in the late-time limit $\left(t / t_{L} \gg 1\right)$, the exponent $\beta$ is expected to converge to the theoretical value $1 / 2$ (Kowal \& Worster 2015). We note that these similarity solutions are valid when the thin-film approximation is satisfied, i.e. when $r_{i} / h_{i} \gtrsim 10$, where $h_{i}$ is the characteristic thickness of each fluid layer. We estimate each of these ratios by assuming that the instantaneous fluid volume in each layer is distributed as a disc of radius $r_{i}$ and thickness $h_{i}$. Therefore, the thin-film condition becomes $r_{N} / h=\pi r_{N}^{3} / Q t \gtrsim 10$ for the top fluid, and similarly, $r_{L} / h_{\ell}=\pi r_{L}^{3} / Q_{\ell}\left(t-t_{L}\right) \gtrsim$ 10 for the lubricating fluid.

Results of the comparison to non-lubricated GCs and to purely Newtonian lubricated GCs indicate growing discrepancies between our measured position of the fronts with the 


\section{P. Kumar, S. Zuri, D. Kogan, M. Gottlieb and R. Sayag}

existing theories (figure 3c), particularly during the lubricated stage of the flow. Despite the differences, we find that in all the experiments (figure $2 c$ ), both fronts follow a power-law time evolution (figure 4). To quantitatively examine the evolution of the fronts, we first consider the front $r_{N}$ of the non-Newtonian fluid separately during the non-lubricated interval $\left(t / t_{L}<1\right.$, figure $\left.4 a, b\right)$, and during the lubricated interval $\left(t / t_{L} \geq 1\right.$, figure $\left.4 c\right)$. During the initial non-lubricated interval, we expect the front to evolve consistently with $(3.2 a)$ as soon as the lubrication-approximation condition is satisfied. Fitting a power-law function of the form $(3.2 a)$ for the measured $r_{N}$ of the $1 \%$ solutions, we find an exponent that corresponds to $n_{1} \%=3.2 \pm 0.7$ and an intercept that corresponds to $k_{1} \%=6.2 \pm 0.9 \mathrm{~Pa} \mathrm{~s}^{1 / n}$, which are in close agreement with the independently measured fluid parameters in (2.2). We note that the apparent larger difference in the value of $n_{1} \%$ with (2.2) arises from the form of the time exponent $(2 n+2) /(5 n+3)$, which tends to map small exponent differences to large differences in $n$. More precisely, the exponents corresponding to the rheometer and the GC measurements of the $1 \%$ solutions are respectively 0.424 and 0.444 , which reflects a very close agreement with the theory (figure $4 a$ ). The difference may have originated from the finite equilibration time of the viscosity, as we elaborate in $\S 4$. Similarly, for the $2 \%$ solutions, we find that $n_{2} \%=2.1 \pm 0.5$ and $k_{2} \%=18.1 \pm 7.2 \mathrm{~Pa} \mathrm{~s}^{1 / n}$. Here the exponents corresponding to the rheometer and the GC measurements are respectively 0.42 and $0.46 \pm 0.01$, which reflects a larger difference than the $1 \%$ case (figure $4 b$ ). Moreover, in this case, the measured $n_{2} \%$ is smaller than $n_{1} \%$ in contrast with the expected growth of $n$ with concentration that was implied in the rheometer measurement (2.2), which suggests that other effects may be present in addition to finite equilibrium time of the viscosity. Our preliminary experiments (figures $2 a$ and $4 a, b$ ) indicate no measurable sensitivity to the substrate wetting conditions or the substrate material (glass or acrylic). Therefore, we propose that wall slip may arise at high polymer concentrations, as we elaborate in $\S 4$.

During the lubricated interval $\left(t / t_{L} \geq 1\right)$, we measure a faster propagation of $r_{N}$ than a non-lubricated GC (figure $4 c$ ), which implies a significant impact of lubrication. The response of $r_{N}$ to the lubrication fluid initialises with a transient acceleration $(1.5 \lesssim$ $t / t_{L} \lesssim 5$ ) with respect to the non-lubricated solution, then during $5 \lesssim t / t_{L}$, the front has a power-law evolution with a similar exponent as that predicted for non-lubricated GCs, but with a larger intercept. Specifically, we find that the time exponent based on the GC experiments is $1.01 \pm 0.04$ times the theoretical value $(2 n+2) /(5 n+3)$ of non-lubricated GCs $(3.2 a)$, with the fluid exponents $n$ predicted by the rheological measurements (2.2). Therefore, during the developed lubricated stage, the front of the PL fluid $r_{N}$ propagates faster than that of a corresponding non-lubricated GC, but with the same time exponent within the measurement uncertainty.

The flow of the lubricating fluid was affected by both the normal and shear stresses applied by the surrounding non-Newtonian fluid. Nevertheless, we find that the evolution of $r_{L}$ is power law in time with an exponent $0.5 \pm 0.02$ (figure $4 d$ ), which is identical to, within the measurement uncertainty, the $1 / 2$ exponent predicted for Newtonian GCs (Huppert 1982; Kowal \& Worster 2015). However, the intercept $b_{L}^{*}$ of the purely Newtonian lubricated theory $(3.2 c)$ that we use to normalise the experimental data does not lead to a full collapse of the data (figure $4 d$ ). Therefore, the intercept in the present case may have a different dependence on the flow parameters and in particular, may depend on the 
(a)

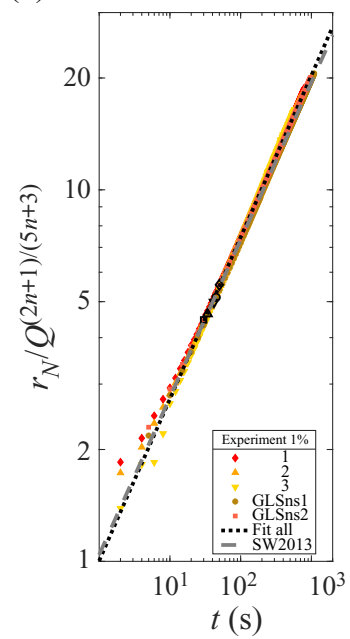

(b)

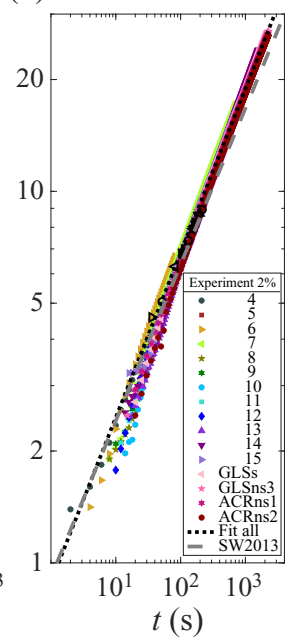

(c)

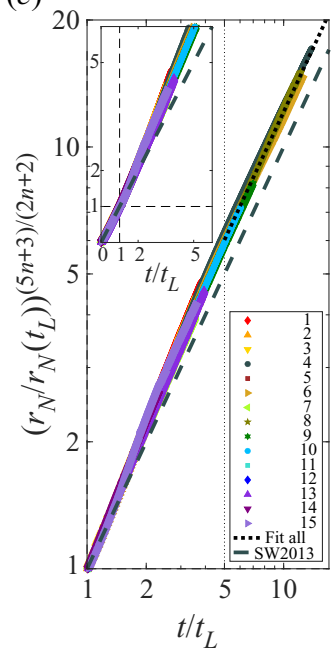

(d)

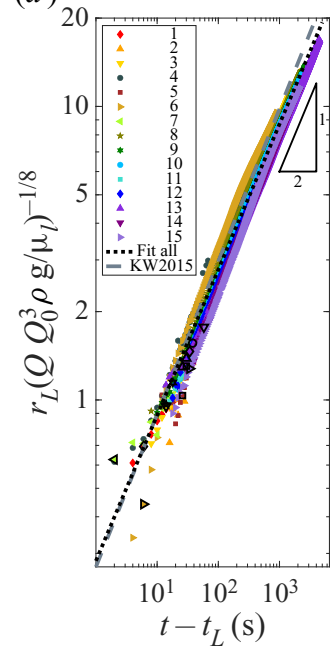

Figure 4. Evolution of the fluid fronts, measurements and theory. $(a, b)$ Measurements of the front $r_{N}(t)$ (markers) during the non-lubricated interval $\left(t / t_{L}<1\right)$ normalised by the flux, for the $1 \%(a)$ and $2 \%(b)$ concentrations. Regression to the experimental measurements $(\cdots$, black) is compared with the theoretical prediction (3.2a) with the fluid parameters measured by the rheometer in $\$ 2.1$ (---, grey). (c) The measured front $r_{N}(t)$ during the lubricated interval $\left(t>t_{L}\right)$, normalised by $r_{N}\left(t=t_{L}\right)$ and by the inverse of the time exponent in (3.2a) with the fluid exponents measured by the rheometer in $\S 2.1$. The normalised front of a non-lubricated GC (3.2a) is shown (---, grey), and is compared with regression to the experimental measurements $(\cdots$, black). Inset zoomed-in image of the earlier stage that includes the non-lubricated interval. $(d)$ The measured front $r_{L}(t)$ (markers) normalised with the coefficient $b_{L}^{*}(3.2 c)$. Regression to the experimental measurements ( $\cdots$, black) is compared with the theoretical prediction (3.2c) of purely Newtonian lubricating GCs (---, grey). The markers when the thin-film criterion is first satisfied have black edges. The STD error bars are too small to be presented.

properties of the non-Newtonian fluid. We also note that for all the experiments (figure $2 c$ )

$$
b_{L} / b_{L}^{*}=\left(\frac{1}{3} \mathscr{D} \mathscr{Q}^{2}\right)^{1 / 8}<1,
$$

which implies that the interaction with the top fluid layer in the purely Newtonian case leads to faster propagation of the lower-layer front $r_{L}$. This appears to be also the response in the present non-Newtonian case, as can be appreciated in figure 3(c).

\subsection{The thickness evolution of the lubricated power-law fluid}

Mass conservation implies that the thickness of lubricated GCs should be smaller than that of non-lubricated GCs to account for the former's faster front propagation. To investigate this, we measured the fluid thickness field from the light intensity extracted from the set of images of the propagating $\mathrm{GC}$ in the method described below.

When a monochromatic light of intensity $I_{0}$ propagates through a fluid layer, the intensity of the transmitted light $I$ drops exponentially with the fluid thickness $h$ according to the Beer-Lambert-Bouguer law (e.g. Bouguer 1729)

$$
h=-\frac{1}{a_{c}} \ln \left(I / I_{0}\right),
$$

where $a_{c}$ is the attenuation coefficient, which we consider as depth independent. We calculate $a_{c}$ using the measured normalised intensity $I / I_{0}$ of a non-lubricated GC together 


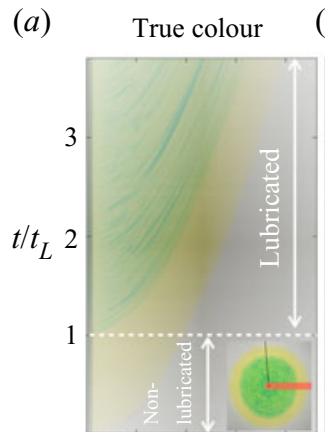

$\begin{array}{llll}10 & 20 & 30 & 40\end{array}$

Radius (cm) (b) Blue channel $I / I_{0}$

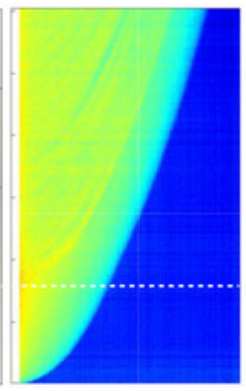

$\begin{array}{llll}10 & 20 & 30 & 40\end{array}$

Radius (cm) (c) Red channel $I / I_{0}$

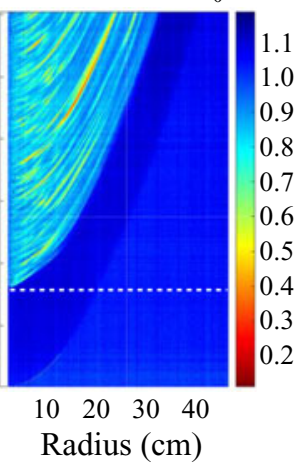

(d)

$t / t_{L}=3.8$

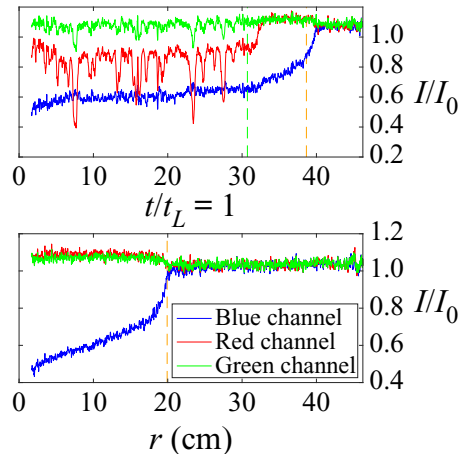

Figure 5. Tracing the light intensity transmitted through the evolving flow. (a) Time series (vertical axis) of snapshots in true colour of the rectangular region along a radius (red region, inset), showing the initial non-lubricated phase $\left(t / t_{L}<1\right)$ in which only the non-Newtonian fluid (yellow) is present, and the lubricated phase $\left(t / t_{L}>1\right)$ in which the lubricated fluid (appears green) GC develops as well. (b) The light intensity captured by the camera's blue channel of the image in $(a)$, normalised by the source intensity $I_{0}$, reveals the thickness distribution of the non-Newtonian fluid at the top layer. (c) The same as in $(b)$ but showing the camera's red channel, which emphasises the distribution of the lubricating fluid. $(d)$ The instantaneous, normalised intensities of the camera's red, green and blue channels along one radius just before the discharge of the lubrication fluid $\left(t / t_{L}=1\right.$, bottom), and some time after $\left(t / t_{L}=3.8\right.$, top).

with the known solution for the thickness of a non-lubricated GC of PL fluid

$$
h=c_{0}(Q, \rho, n) t^{(n-1) /(5 n+3)} \psi\left(r / r_{N}\right),
$$

where $c_{0}$ is a known constant and $\psi$ is the dimensionless solution to a nonlinear differential equation that we solve numerically (Sayag \& Worster 2013). Specifically, we trace the transmitted light intensity along a radius during the non-lubricated phase of the flow, when only the PL fluid is present (figure $5 a$ ) to get the evolution of the normalised light intensity along that radius $I(r, t) / I_{0}$, which consists of red, green and blue (RGB) components defined by the camera's RGB sensor (figure $5 b, c$ ). The yellow dye of the PL fluid absorbs mostly the blue range of wavelengths, as defined by the response spectra of the camera's blue-channel sensor, and hardly attenuates the red and green range of wavelengths (see appendix). Therefore, we expect a growing attenuation of the blue component of the normalised intensity with the non-Newtonian fluid thickness (figure $5 d$ ). Consequently, fitting the normalised transmission of the blue component of $I\left(r, t / t_{L}<1\right) / I_{0}$ to (3.4), where (3.5) is substituted for the thickness (figure $6 a$ ), we obtain the blue light attenuation coefficient $a_{c=\text { blue }} \approx 0.054 \pm 0.0007 \mathrm{~mm}^{-1}$.

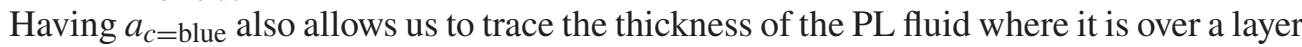
of the lubrication fluid. This can be done even though the light is transmitted through two layers of different fluids because the lubrication fluid is dyed in blue that absorbs mostly in the green-red range and transmits over $96 \%$ of the light intensity in the blue range (see appendix). Therefore, nearly all of the blue-light absorption occurs in the yellow top layer, which implies that we can infer the thickness of the PL fluid throughout the flow using (3.4) with the same coefficient $a_{c=\text { blue }}$.

Results of the conversion of light intensity to thickness (figure $6 b$ ) show that during the lubricated phase of the flow $\left(t / t_{L}>1\right)$, the thickness of the top layer dropped over time with respect to that of the corresponding non-lubricated GC of a similar fluid, as we expect, and became nearly uniform along the entire lubricated part (figure 6b(ii-iv)). 
(a)

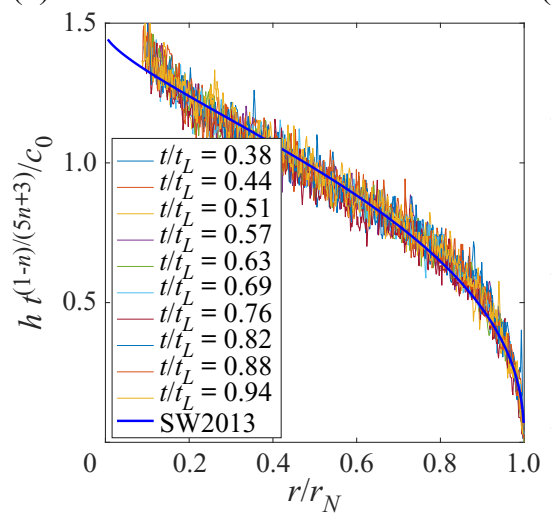

(b)

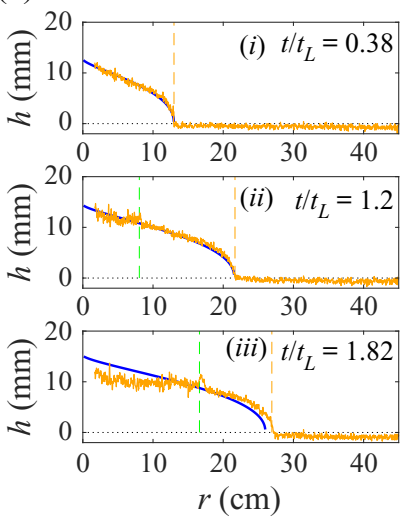

(c)
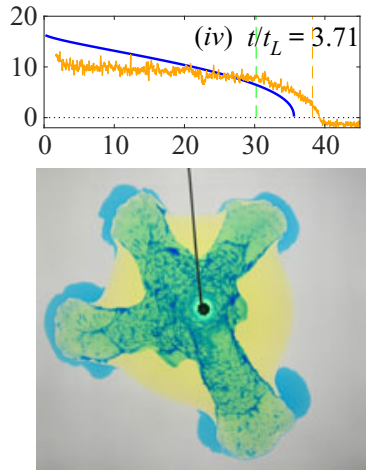

Figure 6. (a) Computation of the blue-light attenuation coefficient $a_{c=b l u e}$ by the regression of selected blue-channel intensity snapshots along a radius during the non-lubricated interval $\left(t / t_{L}<1\right)$ to the known thickness solution (- blue) of a non-lubricated GC of PL fluid (Sayag \& Worster 2013). (b) The thickness field of the top fluid layer resolved from the transmitted light intensity, which shows the thickness along a radius at four different times during the non-lubricated phase (i) and during the lubricated phase (ii-iv) (experiment no. $1, \ldots$, orange), compared with the corresponding solution (Sayag \& Worster 2013) for the thickness of a non-lubricated GC of PL fluid (- blue). Vertical grid lines mark the measured $r_{N}\left(---\right.$, orange) and $r_{L}(---$, green). (c) A snapshot from an experiment with a higher flux ratio $(\mathscr{Q} \approx 0.2, \mathscr{M} \approx 7500)$, which shows the breaking of axisymmetry and the emergence of radial streams.

The thickness of the lubricating layer can be resolved from the red component of the transmitted light, which was absorbed mostly by the blue-dyed lubricating fluid and hardly by the yellow-dyed PL fluid (figure $5 c, d$ and appendix). One possible method to convert the intensity measurements to fluid thickness is by using a priori measurements of known fluid thicknesses (e.g. Vernay, Ramos \& Ligoure 2015). Here we estimate the average thickness of the lubricating film using mass conservation and the known position of the axisymmetric front $r_{L}$. Assuming that the lubricating fluid has a uniform thickness $h_{\ell}$, conservation of mass implies that $h_{\ell}=Q_{\ell}\left(t-t_{L}\right) / \rho_{\ell} \pi r_{L}^{2}$. Using the parameters of experiment no. 1 (figure $2 c$ ) and the measured $r_{L}(t)$, we find that at $t / t_{L}=1.2\left(r_{L}=8.05\right.$ $\mathrm{cm}) h_{\ell} \approx 0.437 \mathrm{~mm}$ (figure 6bii), and at $t / t_{L}=3.71\left(r_{L}=30.21 \mathrm{~cm}\right) h_{\ell} \approx 0.434 \mathrm{~mm}$ (figure 6biv). This implies that the thickness of the lubricating-fluid layer in the experiment was approximately 25 times thinner than that of the PL fluid layer during most of the flow. Therefore, the thickness profiles of the non-Newtonian fluid in figure $6(b)$ represent quite accurately the free surface of the GC.

\section{Discussion}

We assumed that the dominant deformation mechanism of our xanthan solutions in the flow experiments was viscous. To confirm this, we estimate the Debora number $D e=\lambda / T$, where $\lambda \approx 0.1 \mathrm{~s}$ is the elastic relaxation time for $1 \%$ solutions (Stokes et al. 2011) and $T=\pi r^{2} h \rho / Q$ is the characteristic timescale of the flow. Having $h \approx 1 \mathrm{~cm}, Q / \rho \approx 0.5-5$ $\mathrm{cm}^{3} \mathrm{~s}^{-1}$, and using $r_{N}\left(t_{L}\right) \approx 10-20 \mathrm{~cm}$ for the radius (figure $2 c$ ), the smallest timescale we obtain is $T \approx 60 \mathrm{~s}$, which results in $D e \approx 0.0017$ at most and implies that elastic deformation is negligible. The radius where elasticity may have been important $(D e \approx 1)$ is $r \approx 4 \mathrm{~mm}$, which is the radius of the nozzle. The relaxation time of the $2 \%$ solutions is larger than $\lambda$, but even if it is tenfold larger, $D e$ will still be low. 


\section{P. Kumar, S. Zuri, D. Kogan, M. Gottlieb and R. Sayag}

The viscous-PL deformation (2.1) that we assume when analysing the GC experiments is based on the viscosity measurements presented in $\$ 2.1$. The finite shear-rate dependent, viscosity equilibration time that we observed in these measurements (figure $1 c$ ) could potentially imply that the viscosity field in the GC did not have sufficient time to attain the equilibrium value in some regions of the flow. Consequently, the use of (2.1) to represent an instantaneous adjustment of the viscosity to an applied shear rate in the GC may not be strictly valid. However, the good agreement between the theory and experiments in determining the front $r_{N}$ during the non-lubricated flow stage (e.g. figure 4a,b and Sayag \& Worster 2013) suggests that the flow was indeed close to quasi-equilibrium, possibly because the strain-rate field in the GC experiments evolved continuously, in contrast with its step-like variation in the viscosity measurements. Consequently, throughout the flow, the instantaneous viscosity field in the GC remained close to the equilibrium values measured in $\$ 2.1$ (figure 1c). Nevertheless, the small discrepancy that we measure in the initial non-lubricated stage of the GC flow between the measured front $r_{N}$ and the theoretical predictions (figure $4 a, b$ ) could result from the instantaneous viscosity being further away from equilibrium in some regions of the flow. As the viscosity measurements imply $(\$ 2.1)$, the difference between the instantaneous and equilibrium viscosities could peak where the strain rate is smallest. Using the characteristic timescale $T$, we find that the characteristic strain rate in the GC experiments, $T^{-1}$, ranged between $20 / \pi \mathrm{s}^{-1}$ near the nozzle $\left(r=0.5 \mathrm{~cm}, Q / \rho=5 \mathrm{~cm}^{3} \mathrm{~s}^{-1}\right)$ and $0.0016 \mathrm{~s}^{-1}$ at $r=10 \mathrm{~cm}\left(Q / \rho=0.5 \mathrm{~cm}^{3}\right.$ $\left.\mathrm{s}^{-1}\right)$ and $0.0004 \mathrm{~s}^{-1}$ at $r=20 \mathrm{~cm}\left(Q / \rho=0.5 \mathrm{~cm}^{3} \mathrm{~s}^{-1}\right)$. Therefore, we expect that a discrepancy between the equilibrium viscosity and the instantaneous $\mathrm{GC}$ viscosity grows with the radius and inversely with the flux.

Our analysis suggests that the discrepancy between the theoretical predictions (Sayag $\&$ Worster 2013) and the measured front propagation during the non-lubricated interval is larger in the $2 \%$ than in the $1 \%$ concentrations. This discrepancy may be partially explained by the viscosity finite equilibrium time that we discussed previously. Another contribution to this discrepancy may arise from the larger extent of polymer entanglements in the $2 \%$ solution relative to the $1 \%$ solution (the storage modulus of the former is $\sim 3$ times larger than that of the latter, Martín-Alfonso et al. 2018). Consequently, one process that could lead to a faster propagation and be more substantial in the $2 \%$ solutions than the $1 \%$ is wall slip at the fluid-solid interface, potentially through adhesive failure of the polymer chains at the solid surface or through cohesive failure owing to disantanglement of chains in the bulk from chains adsorbed at the wall (Brochard \& Gennes 1992). Such mechanisms would invalidate the theoretically-assumed no-slip condition.

Although we did not calculate the thickness distribution of the lubricating fluid, the true-colour images and the transmitted red-light intensity suggest that it has a complex spatiotemporal structure. In particular, the thickness is non-monotonic, and contains spikes of $50 \%$ reduction in the transmission that appear to propagate downstream with the flow (figure $5 c, d$ ). This structure may arise from the roughness of the non-Newtonian fluid free surface, which may imply the presence of a yield stress. Alternatively, an instability of the fluid-fluid interface could lead to the growth of finite-amplitude thickness spikes. Having calculated the thicknesses of the lubricating and PL fluids, we can propose a possible explanation for the observed difference between the sensitivity of the two fluids to the wetting conditions on the substrate, even though both were aqueous solutions. The largest impact of the wetting conditions on the flow was through capillary forces near the fluid front, where the curvature of the interface of the fluid was largest. The significance of this force should be evaluated with respect to the driving gravitational force through 


\section{Lubricated gravity currents of power-law fluids}

the Bond number $B o=\left(\ell / \lambda_{c}\right)^{2}$, where $\ell$ is a characteristic length scale of the flow, and $\lambda_{c}=\sqrt{\gamma / \rho g}$ is the capillary length of a fluid with density $\rho$ with respect to the air and surface tension coefficient $\gamma$. For both fluids, $\lambda_{c} \approx 2.5 \mathrm{~mm}$, because their densities and surface tension coefficients differ by less than $10 \%$. However, the characteristic scale $\ell$, which most appropriately in this case is represented by the fluid thickness, is equivalent to $h_{\ell} \approx 0.43 \mathrm{~mm}$ for the lubricating layer and approximately to $10 \mathrm{~mm}$ for the polymer solution. Therefore, $B o \approx 16$ for the non-Newtonian fluid implies negligible capillary forces, whereas $B o \approx 0.03$ for the Newtonian lubricating fluid implies a more significant role for capillary forces. This could explain why a coating of soap solution had a significant impact on the front of the lubricating fluid and a negligible impact on the front of the non-Newtonian fluid.

The transition to the lubricated interval coincides with the growth of a non-axisymmetric component of the fronts and translation of the GCs geometric centre. However, as the GC evolved, these quantities appeared to become confined and attenuated (figure $3 d$ ), which implies that the flow remained axisymmetric to the leading order. This apparent stability may be associated with the substantially lower lubrication flux compared with the flux of the top non-Newtonian fluid. Our hypothesis is reinforced by preliminary experiments with larger $\mathscr{Q}$, which demonstrate the breakdown of axisymmetry and the development of finger-like patterns in both the lubricating and top fluids (figure $6 c$ ). In particular, the non-Newtonian fluid propagated as a stream over each of the apparent fingers in the lubricating fluid, which carried most of the flux, while in the non-lubricated regions located in between the streams, the non-Newtonian fluid was nearly stagnant. Therefore, we expect maximal horizontal shear rates along the margins of the stream and correspondingly minimal viscosity. Such a mechanism, which is absent in the purely Newtonian case (Kowal \& Worster 2019), may be important to sustaining the stream pattern. The formation of these patterns, which are reminiscent to ice streams, will be the subject of our future theoretical and experimental work.

\section{Conclusions}

Lubricated GCs are controlled by complex interactions between two fluid layers. The lower lubricating layer modifies the friction between the substrate and the top layer, which in turn applies stresses that affect the distribution of the lubricating layer. The resulting flow can vary dramatically from non-lubricated GCs and from purely Newtonian lubricated GCs.

In our experiments at a lower flux ratio $(\mathscr{Q}<0.06)$ than in previous studies, we find that the two fronts follow a power-law evolution with different exponents that are similar to their corresponding non-lubricated GCs. Nevertheless, the fronts propagate faster than those of non-lubricated GCs. Specifically, following a transient acceleration during the initial lubricated stage, the front $r_{N}$ of the non-Newtonian fluid evolves with the same exponent $(2 n+2) /(5 n+3)$ as a non-lubricated PL fluid, but with a larger intercept. The larger intercept implies that a lubricated GC of such a non-Newtonian fluid propagates faster than a non-lubricated GC of a similar fluid. Compared with the Newtonian case that has an exponent $1 / 2$, strain-rate softening fluids $(n>1)$ have a smaller exponent, which implies that, asymptotically in time, the front of a strain-rate softening fluid propagates slower than that of a purely Newtonian lubricated GC and that consequently, the thickness of the non-Newtonian fluid is larger. The front $r_{L}$ of the lower lubricating fluid evolves with the same exponent $1 / 2$ as purely Newtonian GCs, but with a larger intercept that appears sensitive to the properties of the overlaying non-Newtonian fluid. Our measurements of the transmitted light intensity allow us to compute the radial distribution of the thickness 
of the non-Newtonian fluid layer. We find that, in the lubricated region, the thickness is nearly uniform, in contrast with the monotonically declining thickness of non-lubricated GCs (Sayag \& Worster 2013), but consistently with the theoretical prediction for the case of Newtonian lubricated GC (Kowal \& Worster 2015). Similar measurements of the lubricating fluid reveal a nonmonotonic thickness distribution with localised spikes, which is not observed in the case of purely Newtonian lubricated GCs.

Despite these complex thickness distributions, the fronts of the two fluids remain axisymmetric throughout the flow, with a weak non-axisymmetric component that relaxes with time or remaines confined. However, preliminary experiments with $\mathscr{Q}>0.06$ reveal strong symmetry breaking of the circular fronts and a transition to a stream-like flow, which implies that for a sufficiently large $\mathscr{Q}$, the axisymmetric fronts can become unstable.

The experiments and analyses we present may provide new insights into the rich phenomenology in potentially similar dynamical systems such as ice-sheet systems coupled with hydrological networks. Future research will focus on theoretical analysis of the lubricated flow considered here, by modeling a GC of a PL fluid propagating over a GC of a Newtonian fluid and investigating the resulting axisymmetric flow when the volumes of the two fluids grow in a power law with time. Further research will explore the breaking of circular symmetry and the emergence of radial streams.

Supplementary movies. Supplementary movies are available at https://doi.org/10.1017/jfm.2021.240.

Acknowledgements. We thank D. Bokobza, S. Kabalo and V. Melnichak for their assistance in setting up the experimental apparatus, and to Jungbunzlauer for the xanthan gum.

Funding. This research was supported by the GERMAN-ISRAELI FOUNDATION (grant No. I240430182015).

Declaration of interests. The authors report no conflict of interest.
Author ORCIDs.
(ㄱ) P. Kumar https://orcid.org/0000-0001-8701-4760;
(1) M. Gottlieb https://orcid.org/0000-0003-2137-3386;
(ㄷ) R. Sayag https://orcid.org/0000-0002-1672-982X.

\section{Appendix. Spectral distribution of the light absorbed by and transmitted through the experimental fluids}

We used the light intensity captured by the red-green-blue (RGB) sensor of a Nikon D5500 camera to resolve the thickness of the fluid layers. To measure the spectral distribution of the light source and of the light absorbed by and transmitted through the PL and Newtonian fluids in the flow experiments, we used an AVANTES spectrometer (wavelength range 200-1100 $\mathrm{nm}$ ). The spectral measurements were performed by positioning the edge of the sensing optical fibre at the same position of the camera that was documenting the flow experiments. Therefore, the light we measured went through the same optical path as in the flow experiments, which included transmission through the glass surface and reflection from the $45^{\circ}$ mirror (figure $1 a$ ). To measure the spectrum of the fluids, we placed each fluid in a Petri dish and placed it over the glass substrate. The thickness of the non-Newtonian polymer solution (dyed yellow) in one dish was $8 \mathrm{~mm}$ and the thickness of the Newtonian glucose solution (dyed blue) in the other dish was $2 \mathrm{~mm}$. The rest of the glass surface was covered with optically opaque fabric, so that only light transmitted through the examined Petri dish reached the spectrometer sensor. 
(a)

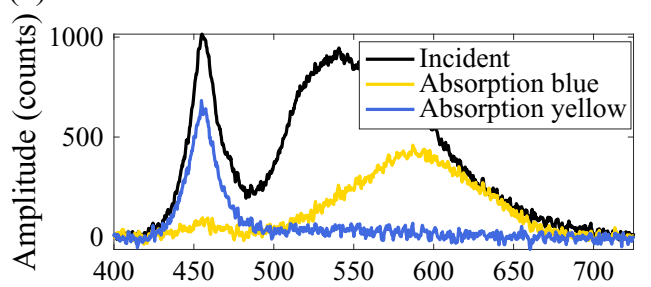

(c)

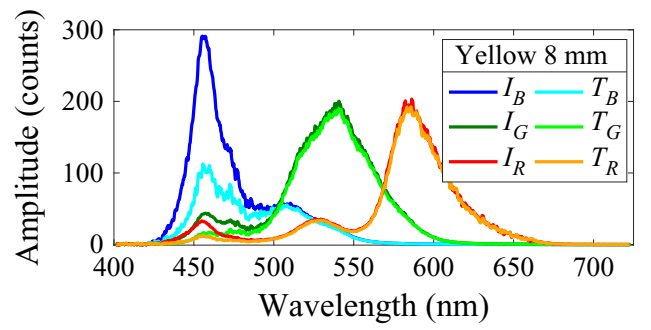

(b)

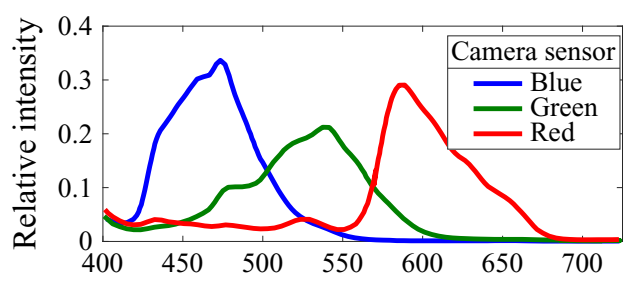

(d)

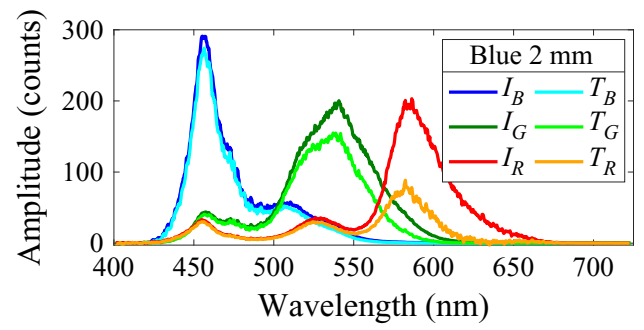

Figure 7. Spectral distributions of the absorbed and transmitted light through the experimental fluids. (a) The spectral distribution of the light-sheet source (- black), and the resulting absorption spectrum of the blue lubricating fluid (- gold) and of the yellow polymer fluid (- blue). (b) Spectral response of the camera's red, blue and green (RGB) channels (Nikon D5000). (c) Spectral distributions of the source incident light on the camera's RGB sensor $I_{R}\left(-\right.$, red), $I_{G}$ (- green) and $I_{B}(-$, blue), compared with the distribution of light transmitted through an $8 \mathrm{~mm}$ thick layer of the yellow polymer fluid on the camera's RGB sensor $T_{R}$ (- orange), $T_{G}$ (- light green) and $T_{B}$ (- cyan). (d) Same distribution as in (c), but comparing the light source with the transmitted light through a $2 \mathrm{~mm}$ thick layer of the blue lubricating fluid.

We find that the light sheet that was used as a source in the flow experiments provided a continuous incident spectrum from violet $\approx 425 \mathrm{~nm}$ to red $\approx 700 \mathrm{~nm}$ (figure $7 a$ ). The blue glucose solution absorbed mostly in the green-red wavelength range (500-700 $\mathrm{nm})$, and the yellow polymer solution absorbed in the blue-violet range $(\lesssim 500 \mathrm{~nm})$ (figure 7a). To evaluate the spectral response of each of the three channels of the camera's RGB sensor to the light signal, we use the spectral response of a Nikon D5000 camera (Bongiorno et al. 2013), which has a similar sensor as the D5500 that we used (figure $7 b$ ). The spectral response of the three-channeled RGB sensor to the light transmitted through our yellow PL fluid (figure $7 c$ ) is substantial in the blue channel and very weak in the green and red channels, which indicates that the light intensity measured by the blue channel provides a leading-order measurement for the yellow-fluid thickness. Similarly, the spectral response of the camera sensor to the light transmitted through our blue solution (figure $7 d$ ) is substantial in the red and green channels and very weak in the blue channel, which indicates that the light intensity measured by the red and green channels provide a leading-order measurement for the blue-fluid thickness. Therefore, light that was transmitted through both fluid layers will have a nearly identical blue-channel spectral distribution as that of a single layer of yellow fluid, which implies that the blue channel provides a measure for the PL fluid thickness throughout the flow.

\section{REFERENCES}

Balmforth, N.J., Burbidge, A.S., Craster, R.V., Salzig, J. \& Shen, A. 2000 Visco-plastic models of isothermal lava domes. J. Fluid Mech. 403, 37-65. 


\section{P. Kumar, S. Zuri, D. Kogan, M. Gottlieb and R. Sayag}

Bongiorno, D.L., Bryson, M., Dansereau, D.G. \& Williams, S.B. 2013 Spectral characterization of COTS RGB cameras using a linear variable edge filter. In Digital Photography IX (ed. N. Sampat \& S. Battiato). SPIE.

Bouguer, P. 1729 Essai d'optique, sur la gradation de la lumière. C. Jombert.

Brochard, F. \& GenNeS, P.G.D. 1992 Shear-dependent slippage at a polymer/solid interface. Langmuir 8 (12), 3033-3037.

Dauck, T.F., Box, F., Gell, L., Neufeld, J.A. \& Lister, J.R. 2019 Shock formation in two-layer equal-density viscous gravity currents. J. Fluid Mech. 863, 730-756.

DeConto, R.M. \& Pollard, D. 2016 Contribution of Antarctica to past and future sea-level rise. Nature 531 (7596), 591-597.

Fowler, A.C. 1981 A theoretical treatment of the sliding of glaciers in the absense of cavitation. Phil. Trans. R. Soc. Lond. A 298 (1445), 637-681.

Fowler, A.C. 1987 A theory of glacier surges. J. Geophys. Res. 92 (B9), 9111-9120.

Griffiths, R.W. 2000 The dynamics of lava flows. Annu. Rev. Fluid Mech. 32, 477-518.

HupPERT, H.E. 1982 The propagation of two-dimensional and axisymmetric viscous gravity currents over a rigid horizontal surface. J. Fluid Mech. 121, 43-58.

Keiser, A., Keiser, L., Clanet, C. \& Quéré, D. 2017 Drop friction on liquid-infused materials. Soft Matter 13 (39), 6981-6987.

Kivelson, M.G., Khurana, K.K., Russell, C.T., Volwerk, M., Walker, R.J. \& Zimmer, C. 2000 Galileo magnetometer measurements: A stronger case for a subsurface ocean at Europa. Science 289 (5483), 1340-1343.

Kowal, K.N. \& Worster, M.G. 2015 Lubricated viscous gravity currents. J. Fluid Mech. 766, 626-655.

KowAL, K.N. \& Worster, M.G. 2019 Stability of lubricated viscous gravity currents. Part 2. Global analysis and stabilisation by buoyancy forces. J. Fluid Mech. 871, 1007-1027.

Lister, J.R. \& KERR, R.C. 1989 The propagation of two-dimensional and axisymmetric viscous gravity currents at a fluid interface. J. Fluid Mech. 203, 215-249.

Martín-Alfonso, J.E., CuAdri, A.A., Berta, M. \& Stading, M. 2018 Relation between concentration and shear-extensional rheology properties of xanthan and guar gum solutions. Carbohyd. Polym. 181, 63-70.

Pegler, S.S. \& Worster, M.G. 2012 Dynamics of a viscous layer flowing radially over an inviscid ocean. J. Fluid Mech. 696, 152-174.

SAYAG, R. \& WORSTER, M.G. 2013 Axisymmetric gravity currents of power-law fluids over a rigid horizontal surface. J. Fluid Mech. 716, R5.

SAYAG, R. \& Worster, M.G. 2019 Instability of radially spreading extensional flows. Part 1. Experimental analysis. J. Fluid Mech. 881, 722-738.

Schoof, C. \& Hewitt, I. 2013 Ice-sheet dynamics. Annu. Rev. Fluid Mech. 45 (1), 217-239.

Stokes, C.R., Clark, C.D., Lian, O.B. \& TulaczyK, S. 2007 Ice stream sticky spots: a review of their identification and influence beneath contemporary and palaeo-ice streams. Earth-Sci. Rev. 81 (3-4), 217-249.

Stokes, J.R., Macakova, L., Chojnicka-Paszun, A., De Kruif, C.G. \& De Jongh, H.H.J. 2011 Lubrication, adsorption, and rheology of aqueous polysaccharide solutions. Langmuir 27, 3474-3484.

Vernay, C., Ramos, L. \& Ligoure, C. 2015 Free radially expanding liquid sheet in air: time- and space-resolved measurement of the thickness field. J. Fluid Mech. 764, 428-444.

Woods, A.W. \& MASON, R. 2000 The dynamics of two-layer gravity-driven flows in permeable rock. J. Fluid Mech. 421, 83-114. 